\title{
Homocysteine and Asymmetric Dimethylarginine (ADMA) in Neurological Diseases
}

\author{
Jolanta Dorszewska, Anna Oczkowska, Margarita Lianeri, Wojciech Kozubski \\ Laboratory of Neurobiology, Department of Neurology, Poznan University of Medical Sciences \\ 49 Przybyszewskiego St. 60-355 Poznan, Poland \\ dorszewskaj@yahoo.com \\ Laboratory of Neurobiology, Department of Neurology, Poznan University of Medical Sciences \\ 49 Przybyszewskiego St. 60-355 Poznan, Poland \\ annaoczkowska@gmail.com \\ Laboratory of Neurobiology, Department of Neurology, Poznan University of Medical Sciences \\ 49 Przybyszewskiego St. 60-355 Poznan, Poland \\ margarita.lianeri@gmail.com \\ Chair and Department of Neurology, Poznan University of Medical Sciences \\ 49 Przybyszewskiego St. 60-355 Poznan, Poland \\ wkozubski@ump.edu.pl
}

\begin{abstract}
Homocysteine (Hcy) is formed from methionine (Met) and is distributed in two metabolic pathways: in the process of remethylation to Met and in the process of transsulfuration to cysteine. Hyperhomocysteinemia (HHcy) is a risk factor for cardiovascular and neurological diseases such as: Alzheimer's and Parkinson's diseases, multiple sclerosis, and stroke. Increased Hcy level may lead to endothelial dysfunction due to impaired bioavailability of endothelium-derived nitric oxide (NO). The molecular mechanism decreasing the levels of $\mathrm{NO}$ in HHcy conditions is incompletely understood, but it seems that asymmetric dimethylarginine (ADMA), an endogenous inhibitor of NO synthase, may be a key factor. ADMA is formed from L-arginine by enzymes in the family of protein N-methyltransferases (PRMT) and may undergo hydrolysis to $L$ citrulline and dimethylamine with the participation of dimethylaminohydrolase (DDAH). In pathological conditions such as neurodegenerative diseases, Hcy may lead to increased ADMA concentrations by inhibiting the activity of DDAH. Several drugs, such L-dopa, antiepileptic drugs, and lipid-lowering drugs, may interfere with the metabolic pathways of thiols, leading to an alteration of plasma Hcy and ADMA levels. It seems that administration of L-arginine, in conjunction with $B$ vitamins, to patients with HHcy may be a new method in the treatment of neurodegenerative diseases.
\end{abstract}

\section{Indexing terms/Keywords}

Biothiols; dimethylarginine; pathogenesis; therapy effects

\section{Academic Discipline And Sub-Disciplines}

Neuroscience

\section{SUBJECT CLASSIFICATION}

Neurology Subject Classification

\section{TYPE (METHOD/APPROACH)}

Literary Analysis; Experimental

\section{Council for Innovative Research}

Peer Review Research Publishing System

\section{Journal: Journal of Advances in Chemistry}

\author{
Vol. 6, No. 2 \\ editor@cirworld.com
}

www.cirworld.com, member.cirworld.com 


\section{INTRODUCTION}

Current literature indicates that a change in the concentration of asymmetric dimethylarginine (ADMA) in hyperhomocysteinemic (HHcy) conditions in patients with neurological diseases, such as stroke [1-3], Alzheimer's disease (AD) [4-6], Parkinson's disease (PD) [7], multiple sclerosis (MS) [8-10], and in patients treated with L-dopa, antiepileptic drugs (AEDs) and lipid-lowering drugs. Siniscalchi et al. [11] indicated that fibric acid derivates, such as fenofibrate and cholestyramine, may increase plasma Hcy levels. It has also been observed that statins, that lower the concentration of homocysteine (Hcy) [e.g. simvastatin at a dose of $80 \mathrm{mg} /$ day], may also decrease ADMA levels [12]. The effectiveness of lowering the concentration of ADMA by statins depends both on the type of pharmaceutical formulation, as well as dose amount. Most research indicates that simvastatin, atorvastatin and pravastatin do not reduce the concentration of ADMA, and their mechanism of improvement of endothelial function is associated with decreased levels of other cardiovascular factors. However, the study by Lu et al. [13] on rosuvastatin and the research by Oguz and Uzunlulu [14] on fluvastatin indicate that a dose of $80 \mathrm{mg} /$ day of either statin for 6 weeks leads to lower concentrations of ADMA.

\section{METABOLISM OF HOMOCYSTEINE AND ADMA}

Homocysteine is formed from methionine (Met) and is distributed in two metabolic pathways: in the processes of remethylation and transsulfuration. Remethylation of Hcy to methionine (Met) may be re-converted to Hcy using Sadenosylmethionine (SAM) and S-adenosylhomocysteine (SAH) or transsulfurated to cysteine (Cys) using cystathionine $\beta$-synthase (CBS).

A product of Met demethylation, SAM is considered to be the main donor of methyl groups in numerous metabolic processes, leading to the formation of methyl derivatives. It is believed that one of the products of the methylation of SAM is ADMA [15].

ADMA, also known as dimethylargininase, is an endogenous inhibitor of nitric oxide (NO) synthase (NOS). ADMA is formed from L-arginine (Arg), contained in body proteins, by enzymes of the family of the protein arginine $\mathrm{N}$ methyltransferases (PRMT) [16]. There are various known methyltransferase proteins, differing by their location in the human body, their products, and the specificity of their substrate. It has been shown that ADMA is a product of PRMT type I, symmetric dimethylarginine (SDMA) is a product of PRMT type II, and monomethylarginine (MMA) is a product of both PRMT type I and II [15]. ADMA can undergo hydrolysis to L-citrulline and dimethylamine with the participation of dimethylaminohydrolase (DDAH) [17]. There are two known forms of the enzyme DDAH, namely DDAH I and DDAH II. DDAH I occurs in cells that express neuronal NOS (nNOS) whereas DDAH II is found in cells with endothelial NOS (eNOS) [16]. However, Hcy increases ADMA concentrations by inhibiting the activity of DDAH, and furthermore can lead to a decrease in the production of NO and L-citrulline from Arg with the participation of NOS (see Figure 1).

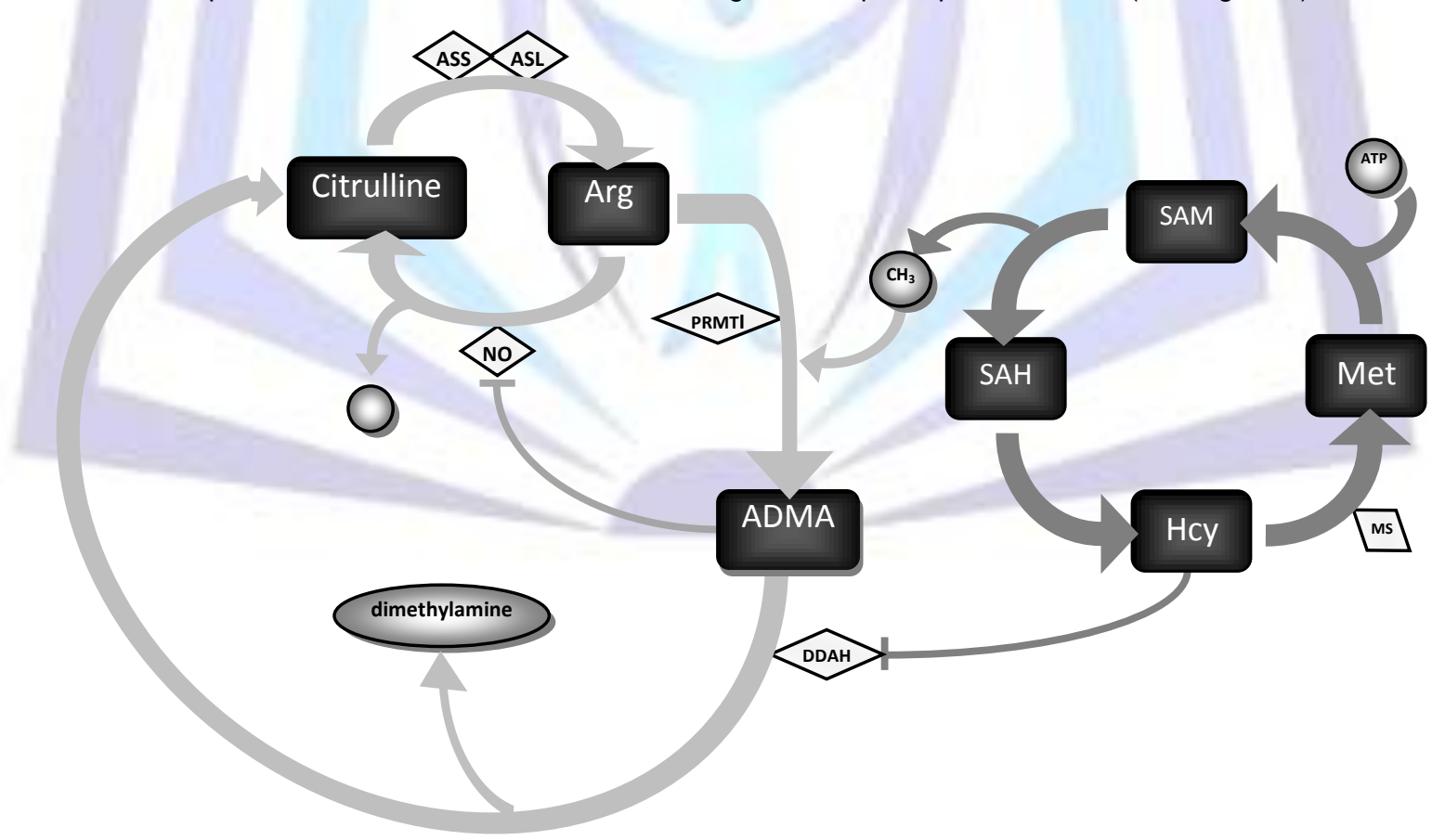

Fig 1: The metabolism of homocysteine and asymmetric dimethylarginine.

Hcy- homocysteine, Met- methionine, ADMA- asymmetric dimethylarginine, Arg- arginine, NO- nitric oxide, NOSnitric oxide synthase, DDAH- dimethylarginine dimethylaminohydrolase, SAM- S-adenosylmethionine, SAH- Sadenosylhomocysteine, PRMT- protein N-methyltransferase, MS- methionine synthase, ASS- argininosuccinate synthase, ASL- argininosuccinate lyase. 


\section{HOMOCYSTEINE, ADMA, AND ISCHEMIC STROKE}

Studies conducted in industrialized countries have shown that levels of both Hcy and ADMA correlate with the risk of atherosclerosis and cardiovascular disease [18]. Furthermore, it has been shown that elevated levels of both Hcy and ADMA in the prooxidative mechanism may lead to vascular endothelial dysfunction, increase smooth muscle cell proliferation, and interfere with platelet function and adhesion of monocytes (see Figure 2) [19].

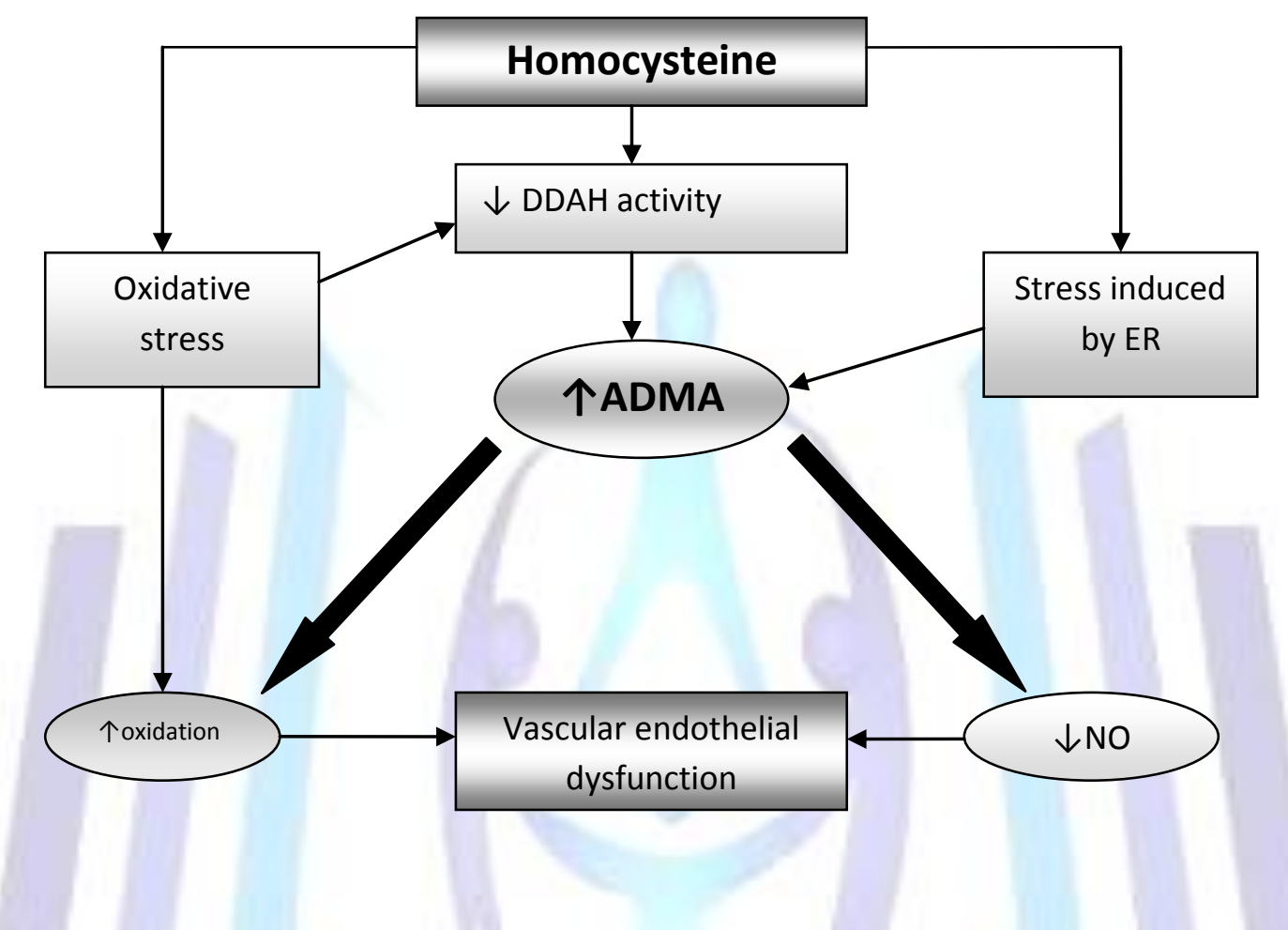

Fig 2: Possible mechanism of vascular endothelial damage involving homocysteine and asymmetric dimethylamine.

ADMA- asymmetric dimethylarginine, DDAH- dimethylarginine dimethylaminohydrolase, NO- nitric oxide, ERendoplasmic reticulum; marked with arrows $\uparrow$ increase, $\downarrow$ decrease indicates the type of influence the parameters have on the concentration of vascular endothelial function.

In the process of aging and vascular diseases associated with old age (including ischemic stroke), vascular endothelial dysfunction involving the $\mathrm{NO}$ formed from Arg, a substrate for the biosynthesis of ADMA, and reduced NO bioavailability are important factors (see Figure 3). A less well-known interaction between Hcy and ADMA, with the development of stroke has been found in patients with stroke, and the possibility of regulating the levels of Hcy and ADMA has been considered in the prevention and treatment of stroke. The study by Yoo and Lee [1] conducted on a Korean population shows an increase in plasma ADMA concentrations, which also positively correlated with Hcy levels, in patients with ischemic stroke - patients aged about 70 years. In addition, the study demonstrated that elevated levels of ADMA are a risk factor for ischemic stroke in the elderly, but only for patients with HHcy in the mechanism associated with renal vasodilatation, and a risk factor of thrombosis in atherosclerotic patients. An increase in plasma ADMA levels has also been found in young patients with ischemic stroke - aged between 15 and 50 years of age [2]. However, multivariate regression analysis including Hcy levels in these patients indicated that Hcy is an independent predictor of elevated plasma ADMA concentrations in stroke occurring around the age of 40 years. Nonetheless, the studies that RuedaClausen et al. [3] conducted in a Spanish population (238 patients and 238 controls, aged between 58.1 and 77.0 years) of stroke sufferers, but with normal renal function, showed that only the level of plasma Hcy, and not ADMA, correlates with the onset of ischemic stroke. The lack of correlation between Hcy and ADMA in this group can be explained on the basis of one of the mechanisms outlining the relationship between these cardiovascular risk factors. It is expected that the reduction in renal excretion of ADMA observed in patients with HHcy, is the result of impaired renal function due to elevated levels of creatinine, which often appears in subjects with elevated plasma Hcy.

It is believed that the effect of Hcy and ADMA in the development of stroke can be treated as a multifactorial mechanism and requires a search for effective therapies aiming to reduce the level of ADMA, a risk factor for stroke, e.g. 
by administration of vitamin $\mathrm{E}$. It has been shown that vitamin $\mathrm{E}$ reduces the levels of ADMA in patients with chronic kidney disease [20].

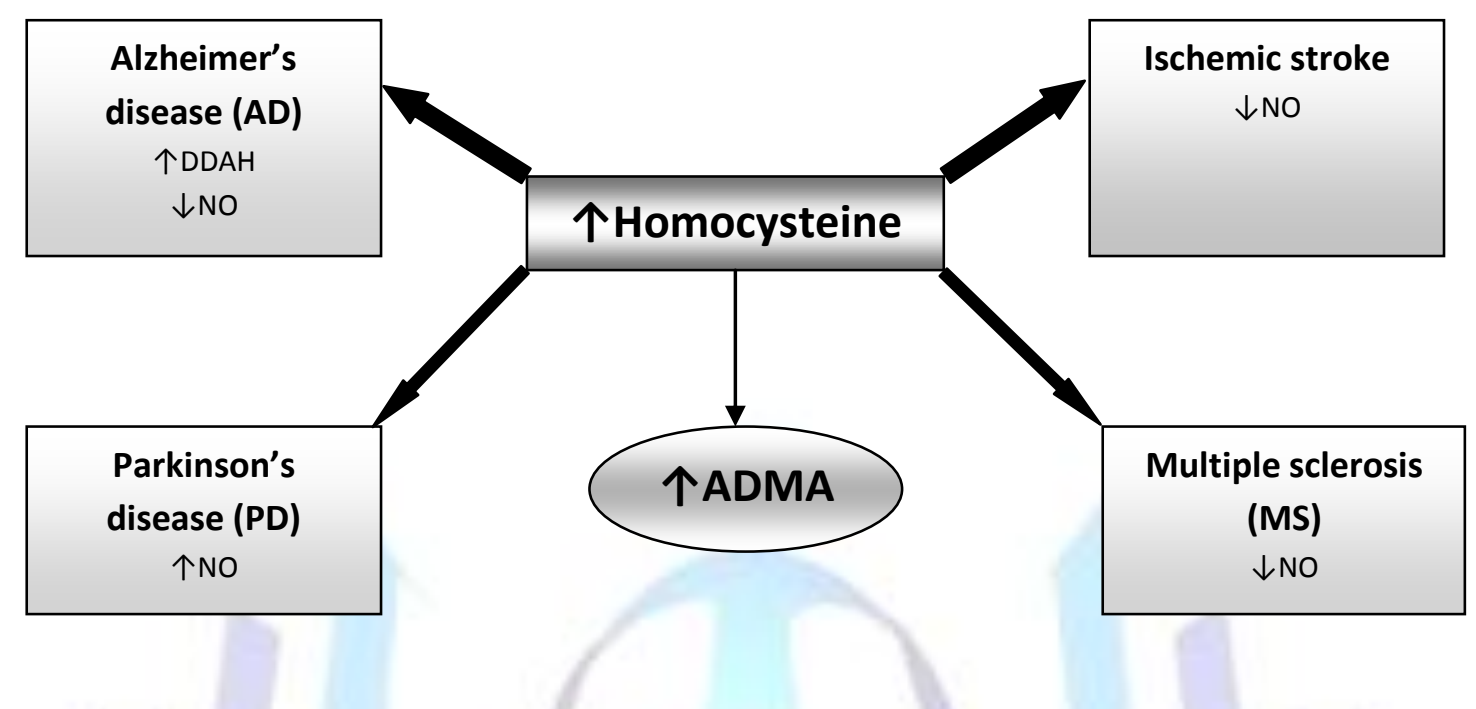

Fig 3: Homocysteine and asymmetric dimethylarginine in neurological diseases.

ADMA- asymmetric dimethylarginine, DDAH- dimethylarginine dimethylaminohydrolase, NO- nitric oxide; marked with arrows $\uparrow$ increase, $\downarrow$ decrease the level of biochemical parameters.

\section{HOMOCYSTEINE AND ADMA IN NORMAL AGING AND ALZHEIMER'S DISEASE}

Homocysteine is considered to be a risk factor for vascular and degenerative diseases [21]. Its elevated levels in the CNS can lead to vascular endothelial damage, impaired NO production and the neurotoxicity seen both in aging and in some degenerative diseases, including AD. The studies of Dorszewska et al. [6,22] have shown that an increased concentration of Hcy in normal aging occurs after 60 years of age it is most likely due to the lowering of Hcy metabolism cofactors as a result of physiological weakening of the body's defense and repair mechanisms. The studies of Dorszewska et al. [6] have also shown that increased concentrations of Hcy can be generated by developing a degenerative disease, and in $A D$ is probably related to a lack of control over the action of presenilin 1 (PS1) [23].

The studies of Dorszewska et al. [6,22] have also shown that an increased concentration of Hcy in AD patients is most likely related to the intensity of the characteristics of dementia in the Mini-Mental State Examination (MMSE) Scale, because patients with $A D$ were observed to have almost double the levels of this sulfur amino acid in the final stages of development of dementia.

It is believed that Hcy in the body is broken down in two main metabolic pathways, through transsulfuration and remethylation (to Met). The studies of Dorszewska et al. [6,22] have shown that the reduction in levels of Met and the Met to Hcy ratio, both in normal aging and in degenerative diseases (e.g. AD), is most likely due to an abnormal remethylation process of Hcy. One of the newer theories concurrently considers that the low levels of Met to Hcy ratio may be associated with an induction of atherosclerosis by Hcy with thiolactone participation [24].

It has been shown that ADMA in patients with HHcy can lead to vascular endothelial dysfunction (see Figure 3) [25]. It is also known that Hcy regulates the concentration of ADMA by blocking the DDAH enzyme. Inhibition of DDAH enzyme activity with Hcy participation leading to an increased concentration of ADMA has been shown in cultures of rat neurons [4], and an increase in DDAH activity has been shown in brain neurons of AD patients [26].

Reports in the literature and the studies of Dorszewska et al. [6,22] have shown that, both in the aging body as well as in AD, there is an increase in plasma concentrations of ADMA [5]. It is believed that, during the aging process, elevated levels of ADMA contribute to reduced bioavailability of oxygen in vascular endothelium, and may be responsible for deterioration of renal excretory function and an increase in blood pressure in the elderly.

However, it is believed that increased concentrations of ADMA in AD patients may contribute to the deterioration of cerebral circulation through a decrease in NO production, increased aggregation and adhesion of leukocytes, vascular endothelial dysfunction and development of atherosclerosis [27]. The deterioration of vascular endothelial function is an important risk factor for $A D$ and may affect the development of dementia. Furthermore, it is believed that the deterioration of cerebral circulation in $A D$ may also affect metabolic disorders, contribute to cognitive function impairment, and aggravate degenerative changes in the CNS $[27,28]$. It is also known that ADMA is considered a risk factor for vascular 
disease and may lead to hypercholesterolemia, hypertension and stroke. All these disorders are considered risk factors for $A D$. Thus, increased levels of ADMA may contribute to the development of $A D$ via atherosclerosis and stroke events. Moreover, studies by Dorszewska et al. [6,22] have observed a downward trend in both concentrations of ADMA and its precursor Arg, responsible for generating NO, during the development of dementia. It is possible that level of NO are increased during the development of $A D$, and oxidation of this NO may form reactive derivatives that are actively involved in the degenerative process. The studies of Chen et al. [29] in patients with AD also showed lower levels of ADMA in the cerebrospinal fluid (CSF), with the development of dementia measuring using the MMSE scale. A reduction in the concentration of ADMA in the CSF was also demonstrated in the study of Arlt et al. [5].

It is believed that low levels of ADMA in the CSF may be associated with increased levels of NO, peroxynitrite production, and damage to macromolecular compounds through oxidative stress, while elevated levels of plasma ADMA may indicate the change in NO metabolism that can lead to a deterioration of cerebral microcirculation.

It seems that both elevated levels of Hcy and ADMA can be regarded as risk factors for AD and may be involved in the pathogenesis of this degenerative disease through the different mechanisms that regulate their levels.

\section{HOMOCYSTEINE, ADMA AND PARKINSON'S DISEASE}

Elevated levels of Hcy are a risk factor for vascular disease and may lead to degenerative diseases, such as PD. Gorell et al. [30] have shown that patients with PD have an increased risk of cardiovascular disease and stroke, not only with the participation of Hcy but also with Cys, the product of Hcy metabolism, through the development of atherosclerosis and thrombosis [31]. It is believed that high levels of Hcy in PD may increase the risk of this neurodegenerative disease by toxic effects in dopaminergic neurons. In vitro studies conducted on human dopaminergic neurons have shown a significant increase in neurotoxicity associated with high Hcy levels [32]. High Hcy concentrations in patients with PD may also lead to impaired cognitive and motor skills and the development of depression.

The study of Dorszewska and Kozubski [7] demonstrated that, in PD patients, an increase in plasma Hcy concentration was accompanied by generation of ADMA. The role of ADMA in the pathogenesis of PD is less known. It has been shown that the level of Arg, a substrate for the biosynthesis of ADMA in CSF, increases during the development of PD. ADMA can result in changes in the level of NO, which has reactive derivatives that can cause oxidative damage to the CNS (see Figure 3).

It is believed that toxic peroxynitrites play a primary role in the development of PD [33]. This seems to confirm the observation that there is no increase in ADMA levels from the second stage of PD, accompanied by decreased levels of Arg that are particularly evident in the fourth stage of the development of PD, that has been demonstrated in the study of Dorszewska and Kozubski [7]. Thus, the participation of reactive NO derivatives is possible in the toxic damage caused to the substantia nigra in PD, and may be controlled by the level of ADMA. Dorszewska and Kozubski [7] have also shown a clear decrease in relative blood Arg to ADMA ratio levels in patients with PD. It is believed that a decrease of this ratio in the blood may be associated with hypercholesterolemia, heart failure, and hypertension.

It also seems that the increased ADMA levels in PD can be influenced by L-dopa treatment. Both the study by Qureshi et al. [34] and that by Dorszewska and Kozubski [7] have been shown a decrease in Arg levels in PD patients treated with this drug. The study by Qureshi et al. [34] also showed that L-dopa therapy produces nitrates, which are neurotoxic factors; however, the work by Dorszewska and Kozubski [7] did not show that ADMA may participate in their generation. Rather, the increased levels of NO seen in PD appear to be the result of increased activity of the glutamatergic system and metabolic changes in neurons [35].

It seems that in development of neurodegenerative diseases, including PD, in which the metabolism of biothiols, i.e. Hcy, Met and Cys is impaired, Hcy and ADMA may be factors involved in disease pathogenesis [7].

\section{HOMOCYSTEINE, ADMA AND MULTIPLE SCLEROSIS (MS)}

Among neurologic diseases, MS belongs to a group of diseases with disorders in the metabolism of biothiols that lead to an increase in Hcy concentration [10]. The study of Triantafyllou et al. [10] showed that increased Hcy levels in patients with MS correlate with symptoms of depression. It is known that depression often occurs in patients with MS and is associated with disease progression in the EDSS (Expanded Disability Status Scale). Furthermore, according to the authors, higher concentrations of Hcy in MS patients with depressive symptoms are associated with low levels of vitamin B12 and folate/folic acid (FA) in most cases. The study by Teunissen et al. [36] demonstrated that the Hcy concentration correlates with disease progression and is higher in patients with secondary progressive MS (SPMS) compared with relapsing-remitting MS (RRMS).

It has been shown that HHcy can induce neuronal damage, leading to the activation of macrophages that is an important factor in the pathogenesis of MS. HyperHcy has also been shown to increase inflammation in the CNS by reducing the production of apolipoprotein $A 1$ (apoA-1). Apo-A1 counteracts inflammation by stimulating the interaction between monocytes and lymphocytes and inhibiting the production of tumor necrosis factor- $\alpha$ (TNF- $\alpha)$ and IL-1 $\beta$, the inflammatory molecules involved in the pathogenesis of MS [37].

In patients with MS, HHcy may also lead to the development of cerebrovascular atherosclerosis and cognitive dysfunction [38]. As demonstrated, cognitive impairment occurs in 30-70\% of MS patients (measured on the PASAT scale, Paced Auditory Serial Addition Test) particularly in patients with the primary progressive MS (PPMS) [36]. According to the authors, further MRI scans of the cerebral cortex of MS patients with HHcy could provide important information on the 
development of cognitive dysfunction in these patients. One of the mechanisms associated with HHcy in MS appears to be a reduced availability of Met, a substrate for the biosynthesis of Hcy with methyl group donor SAM. In the CNS, SAM is involved in numerous methylation reactions and in maintaining neuronal homeostasis, and also affects myelin stability during de- and remethylation.

In the literature there are also a few reports indicating a lack of difference in Hcy levels of patients with MS compared with controls [39].

In MS, HHcy may affect the bioavailability of $\mathrm{NO}$ produced during the formation of nitrosoHcy. ADMA controls the level of NO with the participation of all three NOS isoforms, nNOS, eNOS and induced NOS (iNOS). The study of Masuda and Azuma [9] has shown that, in MS, increased plasma levels of ADMA and endogenous methylarginine, along with disturbances in the biosynthesis of NO may be involved in the pathogenesis of autoimmune disease (see Figure 3). Participation of dimethylarginine in the pathogenesis of MS was also observed in the preliminary study by Rawal et al. [8], where in ADMA excretion in the urine of MS patients (study conducted in 9 patients with MS) shows a decreased trend. Participation of Arg methyl derivatives in the pathogenesis of MS requires further study on a larger group of patients.

\section{HOMOCYSTEINE AND ADMA IN PATIENTS WITH EPILEPSY TREATED WITH ANTIEPILEPTIC DRUGS}

An increase in the concentration of ADMA under conditions of increasing Hcy levels seems to have organic causes [40] and may also be the result of drug therapy [41-43]. The study by Sniezawska et al. [42] shows that epileptic patients treated with antiepileptic drugs (AEDs), have an increased Hcy concentration accompanied by increased concentrations of ADMA, regardless of the type of drugs or polymorphisms of genes encoding enzymes responsible for the efficient metabolism of Hcy to Met.

Studies by Snieżawska et al. [42], (especially during long-term treatment with AEDs) and Jonasson et al. [44], and Wanby et al. [45] conducted in patients with vascular diseases have shown that increases in ADMA concentration do not always result from HHcy. As demonstrated in vascular diseases, a lack in increased ADMA concentration under HHcy conditions may be associated with subtle renal dysfunction in these patients.

Moreover, it has been shown that ADMA may mediate the atherosclerogenic action of Hcy [1]. ADMA is known to be one of the risk factors for cardiovascular disease and markers of atherosclerosis. The pathological effects of Hcy are associated with both the increased levels of ADMA and with a reduced concentration of the metabolite of ADMA and substrate for the production of NO, Arg. According to the study of Snieżawska et al. [42], reduced levels of Arg in patients with epilepsy may be associated with certain genetic predispositions.

Patient with heterozygous AG MTR (A2756G) [methionine synthase] and MTHFD1 GA (G1958A) (methylenetetrahydrofolate dehydrogenase/methylenetetrahydrofolate cyclohydrolase/ formyltetrahydrofolate synthetase) seem to be particularly predisposed to lower levels of this amino acid. It also appears that increased levels of Arg in patients with epilepsy treated with AEDs may be associated with the mechanism of epileptogenesis, in which there is a resultant increase in the biosynthesis of an excitatory (glutamate) and an inhibitory (GABA) neurotransmitter with the participation of this amino acid, or may be the result of mobilization of the body to prevent excessive excitation in the mechanism of action of many AEDs, i.e. increased concentration of GABA [46].

Decreasing of the Arg level in patients with epilepsy also seems to lead to an impaired balance between Arg and ADMA, which according to Dayal and Lentz [47] is most likely the result of increasing oxidative stress associated to increased levels of Hcy. The study of Maas [48] showed that oxidative stress associated with pharmacological intervention results in generation of ADMA by a change in the expression of the genes responsible for synthesis and distribution of this dimethylderivative of Arg. There is literature indicating that there is a change in vascular parameters in epileptic patients treated with AEDs [49].

\section{SUMMARY AND FUTURE PROSPECTS}

Reports in the literature and the results of numerous studies indicate to the involvement of HHcy and ADMA in the pathogenesis of neurological diseases, including stroke, AD, PD, and MS, and in the use of AEDs, L-dopa or lipidlowering drugs. However, there are also studies that have shown no increase in the level of cardiovascular risk factors (Hcy, ADMA) in the development of degenerative disease or resulting from the applied therapy. It also remains unclear share increasing level of ADMA in HHcy as a cause of metabolic changes and an affect renal dysfunction. The impact of $\mathrm{HHcy}$ on the expression of enzymes regulating the levels of ADMA, DDAH and PRMT, in vascular and degenerative diseases is poorly understood. Furthermore, it is not clear if the elevated levels of ADMA are involved in the pathogenesis of endothelial dysfunction in HHcy conditions, as B vitamins and FA treatment to lower ADMA levels in a high concentration of Hcy, as shown in several studies, had no effect. So far, there is also no effective therapy to lower the levels of Hcy and/or ADMA for patients with vascular and degenerative diseases, or those receiving some medications. These issues require further studies. 


\section{REFERENCES}

[1] Yoo, J.H. and Lee, S.C. 2001. Elevated levels of plasma homocyst(e)ine and asymmetric dimethylarginine in elderly patients with stroke. Atherosclerosis 158 (Oct. 2001), 425-430.

[2] Mamatha, S.N., Nagaraja, D., Philip, M. and Christopher, R. 2011. Asymmetric dimethylarginine as a risk marker for early-onset ischemic stroke in Indian population. Clin. Chim. Acta 412 (Jan. 2011), 139-142.

[3] Rueda-Clausen, C.F., Córdoba-Porras, A., Bedoya, G., Silva, F.A., Zarruk, J.G., López-Jaramillo, P. and Villa, L.A. 2012. Increased plasma levels of total homocysteine but not asymmetric dimethylarginine in Hispanic subjects with ischemic stroke FREC-VI sub-study. Eur. J. Neurol. 19 (Mar. 2012), 417-425.

[4] Selley, M.L. 2004. Homocysteine increases the production of asymmetric dimethylarginine in cultured neurons. J. Neurosci. Res. 77 (Jul. 2004), 90-93.

[5] Arlt, S., Schulze, F., Eichenlaub, M., Maas, R., Lehmbeck, J.T., Schwedhelm, E., Jahn, H. and Böger, R.H. 2008. Asymmetrical dimethylarginine is increased in plasma and decreased in cerebrospinal fluid of patients with Alzheimer's disease. Dement. Geriatr. Cogn. Disord. 26, 58-64.

[6] Dorszewska, J., Florczak, J., Jaroszewska-Kolecka, J. and Kozubski W. 2008. Homocysteine and asymmetric dimethylarginine (ADMA) in the plasma of patients with Alzheimer's disease. Folia Neuropathol. 46, 296-297.

[7] Dorszewska, J. and Kozubski, W. 2011. Oxidative DNA damage and the level of biothiols, and L-dopa therapy in Parkinson's disease. In: Qayyum Rana, A. (Ed.) Etiology and Pathophysiology of Parkinson's Disease, InTech, pp. 349-372.

[8] Rawal, N., Lee, Y.J., Whitaker, J.N., Park JO, Paik, W.K. and Kim, S. 1995. Urinary secretion of NG-dimethylarginines in multiple sclerosis patients: preliminary observations. J. Neurol. Sci. 129 (Apr. 1995), 186-191.

[9] Masuda, H. and Azuma, H. 2002. Biological and pathophysiological roles of endogenous methylarginines as inhibitors of nitric oxide synthase. Nihon Yakurigaku Zasshi 119, 29-35.

[10] Triantafyllou, N., Evangelopoulos, M.E., Kimiskidis, V.K., Kararizou, E., Boufidou, F., Fountoulakis, K.N., Siamouli, M., Nikolaou, C., Sfagos, C., Vlaikidis, N. and Vassilopoulos, D. 2008. Increased plasma homocysteine levels in patients with multiple sclerosis and depression. Ann. Gen. Psychiatry 7 (Sep. 2008), 17.

[11] Siniscalchi, A., Mancuso, F., Gallelli, L., Ferreri Ibbadu, G., Biagio Mercuri, N. and De Sarro, G. 2005. Increase in plasma homocysteine levels induced by drug treatments in neurologic patients. Pharmacol. Res. 52 (Nov. 2005), 367375.

[12] McCarty, M.F. 2004. Vascular endothelium is the organ chiefly responsible for the catabolism of plasma asymmetric dimethylarginine-an explanation for the elevation of plasma ADMA in disorders characterized by endothelial dysfunction. Med. Hypotheses 63 ( 2004), 699-708.

[13] Lu, T.M., Ding, Y.A., Leu, H.B., Yin, W.H., Sheu, W.H. and Chu, K.M. 2004. Effect of rosuvastatin on plasma levels of asymmetric dimethylarginine in patients with hypercholesterolemia. Am. J. Cardiol. 94 (Jul. 2004), 157-161.

[14] Oguz, A. and Uzunlulu, M. 2008. Short term fluvastatin treatment lowers serum asymmetric dimethylarginine levels in patients with metabolic syndrome. Int. Heart J. 49 (May 2008), 303-311.

[15] Rho, J., Choi, S., Seong, Y.R., 2001. Prmt5, which forms distinct homo-oligomers, is a member of the protein-arginine methyltransferase family. J. Biol. Chem. 276 (Apr. 2001), 11393-11401.

[16] Leiper, J.M., Santa Maria, J., Chubb, A., MacAllister, R.J., Charles, I.G., Whitley, G.S. and Vallance, P. 1999. Identification of two human dimethylarginine dimethylaminohydrolases with distinct tissue distributions and homology with microbial arginine deiminases. Biochem. J. 343 (Oct. 1999), 209-214.

[17] Ueda, S., Kato, S., Matsuoka, H., Kimoto, M., Okuda, S., Morimatsu, M. and Imaizumi, T. 2003. Regulation of cytokine-induced nitric oxide synthesis by asymmetric dimethylarginine: role of dimethylarginine dimethylaminohydrolase. Circ. Res. 92 (Feb. 2003), 226-33.

[18] Boysen, G., Brander, T., Christensen, H., Gideon, R. and Truelsen, T. 2003. Homocysteine and risk of recurrent stroke. Stroke 34 (May 2003), 1258-1261.

[19] van Guldener, C., Nanayakkara, P.W. and Stehouwer, C.D. 2007. Homocysteine and asymmetric dimethylarginine (ADMA): biochemically linked but differently related to vascular disease in chronic kidney disease. Clin. Chem. Lab. Med. 45, 1683-1687.

[20] Nanayakkara, P.W., Kiefte-de Jong, J.C., ter Wee, P.M., Stehouwer, C.D., van Ittersum, F.J., Olthof, M.R., Teerlink, T., Twisk, J.W., van Guldener, C. and Smulders, Y.M. 2009. Randomized placebo-controlled trial assessing a treatment strategy consisting of pravastatin, vitamin $\mathrm{E}$, and homocysteine lowering on plasma asymmetric dimethylarginine concentration in mild to moderate CKD. Am. J. Kidney Dis. 53 (Jan. 2009), 41-50. 
[21] Seshadri, S., Beiser, A., Selhub, J., Jacques, P.F., Rosenberg, I.H., D'Agostino, R.B., Wilson, P.W. and Wolf, P.A. 2002. Plasma homocysteine as a risk factor for dementia and Alzheimer's disease. N. Engl. J. Med. 346 (Feb. 2002), 476-483.

[22] Dorszewska, J., Florczak-Wyspianska, J., Oczkowska, A., Dezor, M., Prendecki M. and Kozubski W. 2013. Homocysteine and asymmetric dimethylarginine concentrations in the plasma of Alzheimer's disease patients with varying degrees of dementia. Adv. Alzheimers Dis. 1, 1-6.

[23] Fuso, A., Seminara, L., Cavallaro, R.A., D'Anselmi, F. and Scarpa, S. 2005. S-adenosylmethionine/homocysteine cycle alterations modify DNA methylation status with consequent deregulation of PS1 and BACE and beta-amyloid production. Mol. Cell Neurosci. 28 (Jan. 2005), 195-204.

[24] Borowczyk, K., Tisonczyk, J. and Jakubowski, H. 2012. Metabolism and neurotoxicity of homocysteine thiolactone in mice: protective role of bleomycin hydrolase. Amino Acids 43 (Sep. 2012), 1339-1348.

[25] Boger, R.H., Bode-Boger, S.M., Sydow, K., Heistad, D.D. and Lentz, S.R. 2000. Plasma concentration of asymmetric dimethylarginine, an endogenous inhibitor of nitric oxide synthase, is elevated in monkeys with hyperhomocyst(e)inemia or hypercholesterolemia. Arterioscler. Thromb. Vasc. Biol. 20 ( 2000), 1557-1564.

[26] Smith, M.A., Vasák, M., Knipp, M., Castellani, R.J. and Perry, G. 1998. Dimethylargininase, a nitric oxide regulatory protein, in Alzheimer disease. Free Radic. Biol. Med. 25 (Nov. 1998), 898-902.

[27] Selley, M.L. 2003. Increased concentration of homocysteine and asymmetric dimethylarginine and decreased concentrations of nitric oxide in the plasma of patients with Alzheimer's disease. Neurobiol. Aging 24 (Nov. 2003), 903-907.

[28] de la Torre, J.C. 2002. Alzheimer disease as a vascular disorder: nosological evidence. Stroke 33 (Apr. 2002$)$, $1152-$ 1162.

[29] Chen, M., Jiang, P., Lu, J., Xiang, Z.H. and Jiao, B.H. 2010. The correlation of asymmetrical dimethylarginine level and oxidative stress to the onset of Alzheimer's disease. Yao Xue Xue Bao 45 (Aug. 2010), 1001-1005.

[30] Gorell, J.M., Johnson, C.C. and Rybicki, B.A. 1994. Parkinson's disease and its comorbid disorders: an analysis of Michigan mortality data, 1970 to 1990. Neurology 44 (Oct. 1994), 1865-1868.

[31] Muller, T. 2008. Role of homocysteine in the treatment of Parkinson's disease. Expert. Rev. Neurother. 8 ( 2008), 957-967.

[32] Duan, W., Ladenheim, B., Cutler, R.G., Kruman, I.I., Cadet, J.L. and Mattson, M.P. 2002. Dietary folate deficiency and elevated homocysteine levels endanger dopaminergic neurons in models of Parkinson's disease. J. Neurochem. 80 (Jan. 2002), 101-110.

[33] Padovan-Neto, F.E., Echeverry, M.B., Tumas, V. and Del-Bel, E.A. 2009. Nitric oxide synthase inhibition attenuates LDOPA-induced dyskinesias in a rodent model of Parkinson's disease. Neuroscience 159 (Mar. 2009), 927-935.

[34] Qureshi, G.A., Baig, S., Bednar, I., Södersten, P., Forsberg, G. and Siden, A. 1995. Increased cerebrospinal fluid concentration of nitrite in Parkinson's disease. Neuroreport 6 (Aug. 1995), 1642-1644.

[35] Turski, L. and Turski, W.A. 1993. Towards an understanding of the role of glutamate in neurodegenerative disorders: energy metabolism and neuropathology. Experimentia 49 (Dec. 1993), 1064-1072.

[36] Teunissen, C.E., Killestein, J., Kragt, J.J., Polman, C.H., Dijkstra, C.D. and Blom, H.J. 2008. Serum homocysteine levels in relation to clinical progression in multiple sclerosis. J. Neurol. Neurosurg. Psychiatry 79 (Dec. 2008), 13491353.

[37] Hyka, N., Dayer, J.M., Modoux, C., Kohno, T., Edwards, C.K. 3rd, Roux-Lombard, P. and Burger, D. 2001. Apolipoprotein A-I inhibits the production of interleukin-1beta and tumor necrosis factor-alpha by blocking contactmediated activation of monocytes by T lymphocytes. Blood 97 (Apr. 2001), 2381-2389.

[38] Hoffmann, S., Tittgemeyer, M. and von Cramon, D.Y. 2007. Cognitive impairment in multiple sclerosis. Curr. Opin. Neurol. 20 (Jun. 2007), 275-280.

[39] Rio, J., Montalban, J., Tintore, M., Codina, A. and Malinow, M.R. 1994. Serum homocysteine levels in multiple sclerosis. Arch. Neurol. 51 (Dec. 1994), 1181.

[40] Sydow, K., Schwedhelm, E., Arakawa, N., Bode-Böger, S.M., Tsikas, D., Hornig, B., Frölich, J.C. and Böger, R.H. 2003. ADMA and oxidative stress are responsible for endothelial dysfunction in hyperhomocyst(e)inemia: effects of $L$ arginine and B vitamins. Cardiovasc. Res. 57 (Jan. 2003), 244-252.

[41] Oz, O., Gokcil, Z., Bek, S., Cakir, E. and Odabaşi, Z. 2009. Is asymmetric dimethylarginine responsible for the vascular events in patients under antiepileptic drug treatment? Epilepsy Res. 87 (Nov. 2009), 54-58.

[42] Sniezawska, A., Dorszewska, J., Rozycka, A., Przedpelska-Ober, E., Lianeri, M., Jagodziński, P.P. and Kozubski, W. 2011. MTHFR, MTR, and MTHFD1 gene polymorphisms compared to homocysteine and asymmetric dimethylarginine concentrations and their metabolites in epileptic patients treated with antiepileptic drugs. Seizure 20 (Sep. 2011), 533-540. 
[43] Bochynska, A., Lipczynska-Lojkowska, W., Gugala-Iwaniuk, M., Lechowicz, W., Restel, M., Graban, A., Lipska, B. and Gryglewicz, D. 2012. The effect of vitamin B supplementation on homocysteine metabolizm and clinical state of patients with chronic epilepsy treated with carbamazepine and valproic acid. Seizure 21 (May 2011), 276-281.

[44] Jonasson, T.F., Hedner, T., Hultberg, B. and Ohlin, H. 2003. Hyperhomocysteinaemia is not associated with increased levels of asymmetric dimethylarginine in patients with ischaemic heart disease. Eur. J. Clin. Invest. 33 (Jul. 2003), 543-549.

[45] Wanby, P., Brattstrom, L., Brudin, L., Hultberg, B. and Teerlink, T. 2003. Asymmetric dimethylarginine and total homocysteine in plasma after oral methionine loading. Scand. J. Clin. Lab. Invest. 63, 347-353.

[46] Mattson, M.P. 2008. Glutamate and neurotrophic factors in neuronal plasticity and disease. Ann. NY Acad. Sci. 1144 (Nov. 2008), 97-112.

[47] Dayal, S. and Lentz, S.R. 2005. ADMA and hyperhomocysteinemia. Vasc. Med. 10 ( 2005), Suppl 1, 27-33.

[48] Maas, R. 2005. Pharmacotherapies and their influence on asymmetric dimethylarginine (ADMA). Vasc. Med. 10, 4957.

[49] Dorszewska, J., Winczewska-Wiktor, A., Sniezawska, A., Kaczmarek, I. and Steinborn, B. 2009. Homocysteine and asymmetric dimethylarginine (ADMA) in epilepsy. Prz. Lek. 66, 448-452 (in polish).

\section{Author' biography with Photo}

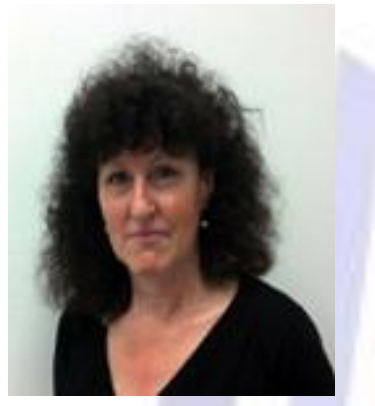

Dr. Jolanta Dorszewska

Laboratory of Neurobiology, Department of Neurology, Poznan University of Medical Sciences, Poznan, Poland

Associate Professor

Chief of Laboratory of Neurobiology

Email: dorszewskaj@yahoo.com

\section{Qualifications}

1996 Ph.D., Poznan University of Medical Sciences, Poznan, Poland, Neurochemistry

1987 M.Sc., Poznan University of Medical Sciences, Poznan, Poland, Pharmacy

\section{Publications}

1. Permoda-Osip A., Dorszewska J., Skibinska M., Chlopocka-Wozniak M., Robakowski J.K. (2013), Hyperhomocysteinemia in bipolar depression: clinical and biochemical correlates, Neuropsychobiology, 68, 193196.

2. Karolczak D., Sawicka E., Dorszewska J., Radel A., Bodnar M., Błaszczyk A., Jagielska J., Marszałek A. (2013), Memantine - neuroprotective drug in aging brain, Pol. J. Pathol., 64, 3, 196-203.

3. Rozycka A., Dorszewska J., Steinborn B., Lianeri M., Winczewska-Wiktor A., Sniezawska A., Wisniewska K., Jagodzinski P.P. (2013), Association Study of the 2-bp Deletion Polymorphism in Exon 6 of the CHRFAM7A Gene with Idiopathic Generalized Epilepsy, DNA Cell Biol., 32, 11, 640-647.

4. Permoda-Osip A., Dorszewska J., Bartkowska-Sniatkowska A., Chlopocka-Wozniak M., Robakowski J.K. (2013), Vitamin B12 level May be related to the efficacy of single ketamine infusion in bipolar depression, Pharmacopsychiatry, 46, 227-228.

5. Dorszewska Jolanta and Kozubski Wojciech (2013). Methionine, homocysteine and cysteine, and antiepileptic drugs in epilepsy. Methionine: Biosynthesis, Chemical Structure and Toxicity, Alexander Snegursky (Ed.), ISBN: 978-1-62618-292-9, NOVA Sciences Publishers, Inc, NY, USA, 155-175.

6. Dorszewska J. (2013), Cell biology of normal brain aging: Synaptic plasticity - Cell death. Aging Clin. Exp. Res., $25,1,25-34$. 
7. Różycka A., Dorszewska J., Steinborn B., Kempisty B., Lianeri M., Wiśniewska K., Jagodziński P.P. (2013), A transcript coding for a partially duplicated form of $\alpha 7$ nicotinic acetylcholine receptor is absent from the CD4+ $T$ lymphocytes of patients with autosomal dominant nocturnal frontal lobe epilepsy (ADNFLE), Folia Neuropathol., $51,1,65-75$.

8. Dorszewska J., Florczak-Wyspiańska J., Oczkowska A., Dezor M., Prendecki M., Kozubski W. (2013), Homocysteine and asymmetric dimethylarginine concentrations in the plasma of Alzheimer's disease patients with varying degrees of dementia, Adv. Alzheimers Dis., 1, 1-6.

9. Dorszewska Jolanta and Kozubski Wojciech (2011). Oxidative DNA Damage and the Level of Biothiols, and LDopa Therapy in Parkinson's Disease, Etiology and Pathophysiology of Parkinson's Disease, Abdul Qayyum Rana (Ed.), ISBN: 978-953-307-462-7, InTech, Available from: http://www.intechopen.com/articles/show/title/oxidative-dna-damage-and-the-level-of-biothiols-and-I-dopatherapy-in-parkinson-s-disease.

10. Sniezawska A., Dorszewska J., Rozycka A., Przedpelska-Ober E., Lianeri M., Jagodzinski P.P, Kozubski W. (2011), MTHFR, MTR, and MTHFD1 gene polymorphisms compared to homocysteine and asymmetric dimethylarginine concentrations and their metabolites in epileptic patients treated with antiepileptic drugs, Seizure, 20, 533-540.

11. Dezor M., Dorszewska J., Florczak J., Kempisty B., Jaroszewska-Kolecka J., Rozycka A., Polrolniczak A., Bugaj R., Jagodzinski P.P., Kozubski W. (2011), Expression of 8-oxoguanine DNA glycosylase 1 (OGG1) and the level of p53 and TNF-alpha proteins in peripheral lymphocytes of patients with Alzheimer's disease, Folia Neuropathol., 49, 123-131.

12. Dorszewska J., Kempisty B., Jaroszewska-Kolecka J., Rózycka A., Florczak J., Lianeri M., Jagodziński P.P., Kozubski W. (2009), Expression and polymorphisms of gene 8-oxoguanine glycosylase 1 and the level of oxidative DNA damage in peripheral blood lymphocytes of patients with Alzheimer's disease, DNA Cell Biol., 28, 11, 579-588.

13. Kaźmierski R., Watała C., Podsiadły E., Dorszewska J., Kozubski W. (2009), Association of atherosclerotic risk factors with carotid adventitial thickness assessed by ultrasonography, J. Clin. Ultrasound, 37, 6, 333-341.

14. Dorszewska J., Florczak J., Różycka A., Kempisty B., Jaroszewska-Kolecka J., Chojnacka K., Trzeciak W.H., Kozubski W. (2007), Oxidative DNA damage and level of thiols as related to polymorphism of MTHFR, MTR, MTHFD1 in Alzheimer's and Parkinson's disease, Acta Neurobiol. Exp., 67, 113-129.

15. Dorszewska J., Florczak J., Różycka A., Jaroszewska-Kolecka J., Trzeciak W.H., Kozubski W. (2005), Polymorphisms of the CHRNA4 gene encoding the a subunit of nicotinic acetylcholine receptor as related to the oxidative DNA damage and the level of apoptotic proteins in lymphocytes of the patients with Alzheimer's disease, DNA Cell Biol., 24, 12, 786-794.

16. Kapelusiak-Pielok M., Adamczewska-Goncerzewicz Z., Dorszewska J., Grochowalska A. (2005), The protective action of alpha-tocopherol on the white matter lipids during moderate hypoxia in rats, Folia Neuropathol., 43, 2, 103-108.

17. Dorszewska J., Adamczewska-Goncerzewicz Z. (2004), Oxidative damage to DNA, p53 gene expression and p53 protein level in the process of aging in rat brain, Respir. Physiol. Neurobiol., 139, 227-236.

18. Dorszewska J., Adamczewska-Goncerzewicz Z., Szczech J. (2004), Apoptotic proteins in the course of aging of central nervous system in the rat, Respir. Physiol. Neurobiol., 139, 145-155.

19. Adamczewska-Goncerzewicz Z., Dorszewska J., Michalak S., Grochowalska A., Moczko J. (2000), The effect of moderate hypoxia on free sterols: content and pattern in white matter, Folia Neuropathol., 38, 35-37. 